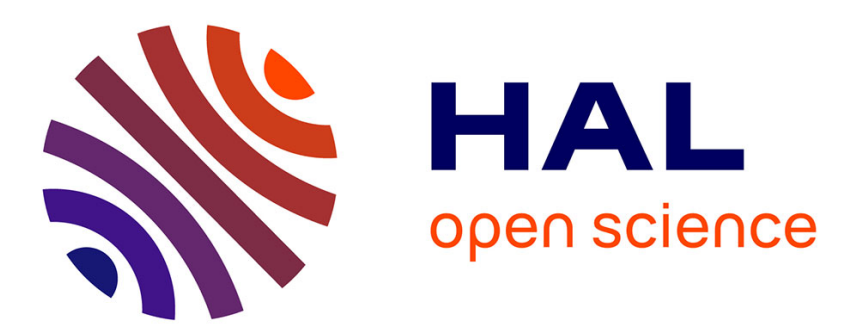

\title{
Complex Non-Linear Modal Analysis for Mechanical Systems: Application to Turbomachinery Bladings With Friction Interfaces
}

Denis Laxalde, Fabrice Thouverez

\section{- To cite this version:}

Denis Laxalde, Fabrice Thouverez. Complex Non-Linear Modal Analysis for Mechanical Systems: Application to Turbomachinery Bladings With Friction Interfaces. Journal of Sound and Vibration, 2009, 322 (4-5), pp.1009-1025. 10.1016/j.jsv.2008.11.044 . hal-00343494v3

\section{HAL Id: hal-00343494 \\ https://hal.science/hal-00343494v3}

Submitted on 4 Jun 2009

HAL is a multi-disciplinary open access archive for the deposit and dissemination of scientific research documents, whether they are published or not. The documents may come from teaching and research institutions in France or abroad, or from public or private research centers.
L'archive ouverte pluridisciplinaire HAL, est destinée au dépôt et à la diffusion de documents scientifiques de niveau recherche, publiés ou non, émanant des établissements d'enseignement et de recherche français ou étrangers, des laboratoires publics ou privés. 


\title{
Complex Non-Linear Modal Analysis for Mechanical Systems: Application to Turbomachinery Bladings With Friction Interfaces
}

\author{
Denis Laxalde*and Fabrice Thouverez \\ Laboratoire de Tribologie et Dynamique des Systèmes UMR-CNRS 5513, \\ École Centrale de Lyon, 36 avenue Guy de Collongue, 69134 Ecully Cedex, France \\ denis.laxalde@ec-lyon.fr, fabrice.thouverez@ec-lyon.fr
}

\begin{abstract}
A method for modal analysis of non-linear and non-conservative mechanical systems is proposed. In particular, dry-friction non-linearities are considered although the method is not restricted to these. Based on the concept of complex non-linear modes, eigensolutions are written as generalized Fourier series and the eigenproblem is then formulated in the frequency-domain. An alternating frequency-time domain method is used for the calculation of implicit non-linear forces. A two degrees-of-freedom example featuring dry-friction illustrates the method and highlights the effects of dissipation on modal parameters. The stabilizing effects of friction in presence of negative damping in the system are also addressed.

Then an application on a large-scale non-linear system consisting of a turbomachinery blade, with dry-friction interfaces is proposed. In the latter, an original framework for the description of two-dimensional frictional motions by complex variables is proposed and applied, in particular, to a Dahl model. Effects of friction parameters and models on the blade's modal characteristics are investigated.
\end{abstract}

Keywords: Non-linear modes; Fourier methods; two-dimensional friction; Dahl model; bladed disks

\section{Introduction}

Non-linear modes provide a mathematical and practical framework for the vibration analysis of non-linear mechanical systems. Theoretical origins of this concept lies in Rosenberg's works [1] while many further developments have then been proposed $[2,3,4,5]$. In this wake, various methods of analysis (e.g., normal form [6], multiple scales [7], averaging [8, 9] or harmonic balance $[3,10,11])$ have been used for the derivation of non-linear normal modes. However, this has undoubtedly received little interest from engineers in the industrial community; very likely because these developments often focus on small-size systems and/or rather academic nonlinearities. There seems to be a consensus on this issue [12] and having recourse to numerical methods for non-linear modal analysis would probably help reducing this gap between academic and industrial communities. Hence, dealing with large-size systems and generic (including nonsmooth) non-linearities appears to be the main challenge of any numerical methods non-linear modal analysis. Recently, several attempts to apply numerical methods to non-linear modal

\footnotetext{
*Present affiliation: Structural Dynamics and Vibration Lab., Department of Mechanical Engineering, McGill University, 817 Sherbrooke street West, Montreal, QC, H3A 2K6, Canada. denis.laxalde@mcgill.ca
} 
analysis have been made. Among others, one can mention a numerical extension of the invariant manifold approach proposed in [13], the class of asymptotic-numerical methods [14, 15] or, recently, a shooting method combined with a continuation procedure [16]. However, the types of non-linearities considered are still mostly limited geometric or polynomial forms, in particular dissipative non-linearities are seldom studied.

This paper addresses this dual issue in a sense that a numerical method for non-linear modal analysis is proposed, which is applicable to large-scale systems and to generic non-conservative non-linearities. First, an original concept of complex non-linear modes is proposed by extension of previous definitions [1, 4] but also by analogy with complex linear modes. This provides a general framework for the treatment of non-linear non-conservative systems (for which the associated autonomous systems are not representative) in terms of vibration modes. Then, the non-linear complex eigenproblem is derived based on a definition of eigenfunctions as generalized Fourier series which fundamental frequency corresponds to the complex eigenvalue. This transformation of the autonomous non-conservative dynamical system's equations of motion from the time-domain to the frequency-domain is done using a Fourier-Galerkin projection. It can also be regarded as a generalization of harmonic balance methods. Motivations for using Fourier methods in the proposed approach rely, in particular, on their ability to deal with non-smooth non-linear functions (see for example [17, 18] or recently [19]). In addition an alternating frequency-time domain scheme [20] is used to transform the non-linear forces in the frequency-domain.

Following the theoretical developments, an academic example, based on a two degreesof-freedom system with a dry-friction non-linearity, is proposed to validate and illustrate the method. Several results and comparisons with time-domain analyses and forced responses are proposed. Also, the potential stabilizing effects of friction damping are studied.

The second example concerns the dynamics of turbomachinery bladings with dry-friction interfaces and illustrates the application of the proposed numerical method on a large-scale system with non-smooth non-linearities. Also, studying the dynamics of such systems in terms of non-linear modes, or more generally in a framework that is different from traditional forced response analyses $[21,22,19]$, can be regarded as a subject in itself. Actually, latter approaches, however efficiently applied in industrial design processes [23, 24, 25], carry fundamental restrictions. First, other types of vibratory phenomena - such as self-excited vibration (synchronous or non-synchronous) and aeroelastic instabilities [26, 27] - cannot be addressed by these forced response approaches. Second, during design processes, assessment of damping performances in contact-friction interfaces is not straightforward with forced response analyses since forcing parameters are generally not exactly known; many parametric studies are often required to achieve a proper design. In this context, modal approaches, such as the one proposed here, are particularly relevant and can help overcoming these issues. Indeed, assessment of friction effects on modal characteristics is straightforward as are parametric analyses. Also, non-linear modal analysis would undoubtedly be suitable in dealing with self-excited vibration phenomena.

Furthermore, insights on friction models and on the treatment of two-dimensional frictional motion are given on this example. First, while the Coulomb model is very popular in many applications, it can sometime be poorly representative of the experimental reality. In this context, more sophisticated models would improve the representativeness of structural dynamics modelling regarding tribological phenomena. A comparison between Coulomb and Dahl [28] models on the considered application is then proposed. Second, the issue of two-dimensional motions in frictional interfaces is tackled. Description of surface motions is proposed using complex variables and this novel approach noticeably simplifies the developments.

The paper is organised into five sections; the two first are dedicated to definitions and formalism of the complex non-linear modal analysis method. The third illustrates it on a two 
degrees-of-freedom example with a friction non-linearity. The fourth section is then dedicated to the afore-mentioned industrial application and precedes concluding remarks.

\section{Complex non-linear modes}

In this first section, a extended definition of non-linear modes is proposed. It is, in essence, related to previous definitions by Rosenberg [1], Szemplinska-Stupnicka [3] or Shaw and Pierre [4] but is also inspired by the definition of complex modes for linear systems. A complex non-linear mode of motion is then defined as an oscillation of the autonomous system with (potentially) a phase difference between its degrees of freedom. This phase difference is the main difference between complex and normal modes since the notion of unison does not appear any more. As second order dynamical systems are considered, the motion takes place on a two-dimensional subspace defined in the system's phase space and is energy-dependent. Finally, and still by analogy with linear complex modes, the eigenvalues of the characteristic equation can be defined in the form

$$
\lambda=-\beta+\mathrm{j} \omega .
$$

In Eq. (1), $\omega=\omega_{0} \sqrt{1-\zeta^{2}}$ is the damped natural angular frequency, $\omega_{0}$ the natural angular frequency and $\zeta=\beta / \omega_{0}$ defines the modal damping ratio.

This completing definition to non-linear modes, which essentially boils down to considering a frequency / damping-energy dependency, appears useful and relevant for practical applications which involve non-linear dissipative systems which cannot be analysed by considering their underlying conservative form. A previous work by the authors [9], in which a Bouc-Wen hysteretic oscillator is studied using an averaging formalism and applied to energy pumping, illustrates the pertinence of this definition.

In the following, a two degrees-of-freedom system with a friction non-linearity (see Fig. 1a) will be considered. Its equations of motion are

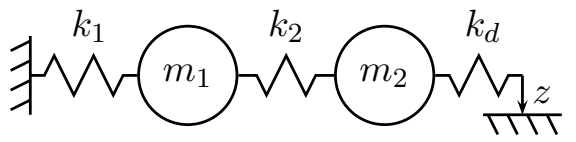

(a)

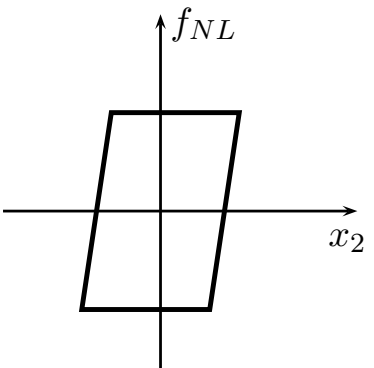

(b)

Figure 1. Guiding example: (a) Oscillator with two degrees of freedom with a friction damper and (b) hysteresis cycle for elastic Coulomb model.

$$
\begin{gathered}
m_{1} \ddot{x}_{1}+k_{1} x_{1}+k_{2}\left(x_{1}-x_{2}\right)=0 \\
m_{2} \ddot{x}_{2}+k_{2}\left(x_{2}-x_{1}\right)+f_{N L}\left(x_{2}, z\right)=0
\end{gathered}
$$

The non-linear restoring force is modelled by means of an elastic Coulomb friction model

$$
f_{N L}=k_{d}\left(x_{2}-z\right)
$$


for which the implicit variable $z$ reprensents the displacement of the (massless) contact point and is calculated in order the force not to exceed the limit Coulomb force $\mu N$. Hence we can introduce an additional differential equation, goverging this friction restoring force:

$$
\dot{z}= \begin{cases}0 & \text { while }\left|f_{N L}\right|<\mu N \\ \dot{x}_{2} & \text { if }\left|f_{N L}\right|=\mu N\end{cases}
$$

An example of hysteresis cycle, representative of this elastic Coulomb model, is shown in Fig. 1b.

Before going further into the methodology of non-linear modal analysis, we put forward a characteristic feature of the non-linear modes of such a dissipative system, which is that trajectories are no longer lines or curves normal to iso-energy curves but closed curves. Fig. 2 shows the trajectories of the system for fixed values of these friction parameters. These responses

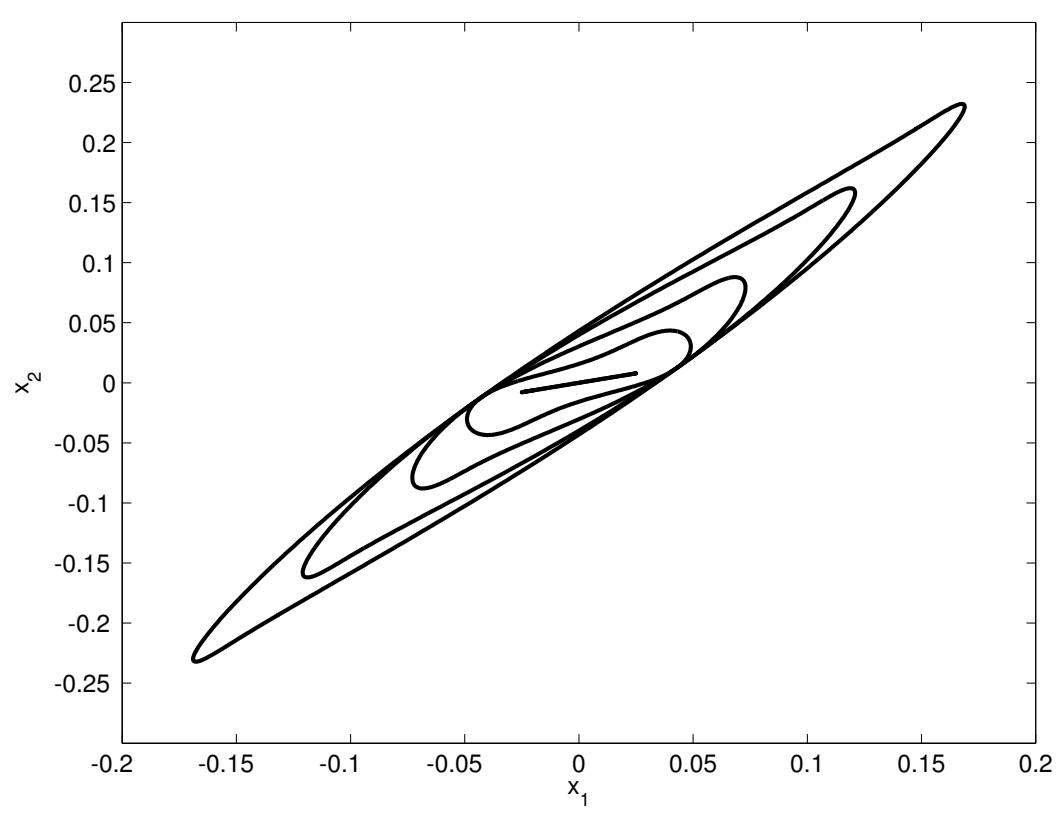

Figure 2. Trajectories of the complex non-linear modes for different amplitudes of vibration

are calculated with the method described beneath. At low levels, the trajectory looks like a straight line due to sticking contact and the system is almost linear. When the energy level increases, non-linear effects appear. These lead to a curvature of the trajectories on the one hand and to their "opening" (because of the non-linear dissipation) on the other hand. A phase difference appears between degrees-of-freedom. These effects cannot be taken into account through the analysis of the conservative system.

\section{Complex non-linear eigenproblem in the frequency-domain}

Following this definition of complex non-linear modes, the complex non-linear eigenproblem will be formulated in the frequency-domain. The developments are based on the definition of eigenfunctions in the form of generalized Fourier series. A Fourier-Galerkin procedure is then naturally used to derived the characteristic equation in the frequency-domain. A generic formulation is obtained which is then reduced by considering systems with frequency-independent 
non-linearities (such as dry-friction). An alternating frequency-time domain scheme is finally used for the derivation of non-linear forces in the frequency domain.

In the following, consider an autonomous dynamical system described by the second order differential equation

$$
\mathbf{m} \ddot{\mathbf{x}}+\mathbf{f}(\mathbf{x}, \dot{\mathbf{x}})=0
$$

where $\mathbf{m}$ is the mass matrix and $\mathbf{f}$ is the vector of non-linear internal forces, dependent on displacements and velocities. Besides conservative terms, this problem includes potentially dissipative terms in $\mathbf{f}$. The purpose is to calculate the modes (in the sense given in section 2) of this non-linear and non-conservative system.

\subsection{Complex eigensolutions as generalized Fourier series}

Given the complex eigenvalue defined by Eq. (1), we define two new time scales $\eta=\beta t$ and $\tau=\omega t$. While the latter is fast and refers to the time scale of oscillations, the first is slow and corresponds their envelope modulation (variations of amplitude) due to non-conservative effects. Then, the complex eigensolutions are expanded as

$$
\mathbf{x}(t) \sim \mathbf{x}(\eta, \tau)=\sum_{p=0}^{\infty} \sum_{n=-\infty}^{\infty} \hat{\mathbf{x}}_{p, n} \mathrm{e}^{-p \eta+\mathrm{j} n \tau} .
$$

The assumed form of Eq. (5) can be regarded as a generalized Fourier series and aims at being representative of damped (i.e. pseudo-periodic) oscillations which naturally occur for autonomous non-conservative systems.

Then, if associated with the inner product

$$
\langle g, h\rangle=\frac{1}{2 \pi} \int_{0}^{\infty} \int_{0}^{2 \pi} g(\eta, \tau) \bar{h}(\eta, \tau) \mathrm{d} \tau \mathrm{d} \eta
$$

the set of functions

$$
\Phi=\left\{\phi_{p, n}(\eta, \tau)=\mathrm{e}^{-p \eta+\mathrm{j} n \tau}:[0, \infty] \times[0,2 \pi] \rightarrow \mathbb{C}\right\}_{p, n=-\infty}^{\infty}
$$

defines an orthogonal base for the class of pseudo-periodic functions. The Fourier components are then given by

$$
\hat{\mathbf{x}}_{p, n}=\frac{\left\langle\mathbf{x}, \phi_{p, n}\right\rangle}{\left\|\phi_{p, n}\right\|^{2}}
$$

The distinction between the two times scales $\eta$ and $\tau$ and their independence in the series (5) enables each oscillating harmonic to have its own rate of slow variation.

The following paragraph concerns the derivation of the complex non-linear eigenproblem associated with this general form of eigensolutions. A degenerated form of eigensolutions and eigenproblem is then introduced for systems with a linear dependency between the two time scales.

\subsection{Derivation of the complex non-linear eigenproblem}

Thanks to Eq. (5), velocities and accelerations are formulated in the frequency-domain

$$
\dot{\mathbf{x}}(t) \sim \dot{\mathbf{x}}(\eta, \tau)=\frac{\partial \mathbf{x}(\eta, \tau)}{\partial \eta} \frac{\mathrm{d} \eta}{\mathrm{d} t}+\frac{\partial \mathbf{x}(\eta, \tau)}{\partial \tau} \frac{\mathrm{d} \tau}{\mathrm{d} t}=\sum_{p=0}^{\infty} \sum_{n=-\infty}^{\infty}(-p \beta+\mathrm{j} n \omega) \hat{\mathbf{x}}_{p, n} \mathrm{e}^{-p \eta+\mathrm{j} n \tau}
$$




$$
\ddot{\mathbf{x}}(t) \sim \ddot{\mathbf{x}}(\eta, \tau)=\frac{\partial \dot{\mathbf{x}}(\eta, \tau)}{\partial \eta} \frac{\mathrm{d} \eta}{\mathrm{d} t}+\frac{\partial \dot{\mathbf{x}}(\eta, \tau)}{\partial \tau} \frac{\mathrm{d} \tau}{\mathrm{d} t}=\sum_{p=0}^{\infty} \sum_{n=-\infty}^{\infty}(-p \beta+\mathrm{j} n \omega)^{2} \hat{\mathbf{x}}_{p, n} \mathrm{e}^{-p \eta+\mathrm{j} n \tau}
$$

Substituting Eqs. (5), (9a) and (9b) in Eq. (4) gives

$$
\mathbf{m} \sum_{p=0}^{\infty} \sum_{n=-\infty}^{\infty}(-p \beta+\mathrm{j} n \omega)^{2} \hat{\mathbf{x}}_{p, n} \phi_{p, n}+\mathbf{f}\left(\sum_{p=0}^{\infty} \sum_{n=-\infty}^{\infty} \hat{\mathbf{x}}_{p, n} \phi_{p, n}, \beta, \omega\right)=0 .
$$

Then, by means of the inner product of Eq. (6), a Fourier-Galerkin projection can be applied to Eq. (10). This leads to the complex frequency-domain weak form of the initial equation of motion:

$$
\forall(p, n) \in \mathbb{N} \times \mathbb{Z}, \quad(-p \beta+\mathrm{j} n \omega)^{2} \mathbf{m} \hat{\mathbf{x}}_{p, n}+\frac{\left\langle\mathbf{f}, \phi_{p, n}\right\rangle}{\left\|\phi_{p, n}\right\|^{2}}=0 .
$$

The algebraic system defined by Eq. (11) is the eigenproblem associated with the proposed definition of complex non-linear modes. Also, while the previous developments consider infinite series in Eqs. (5), (9a) and (9b), an approximation of these is considered for applications by means of truncations: $(p, n) \in\left[0, \ldots, N_{p}\right] \times\left[-N_{n}, \ldots, N_{n}\right]$.

The previous formulation of the eigenproblem is general due to the independence of time scales. We are now interested in the particular class of systems which characteristics of dissipation are independent of the frequency. For such systems, a linear dependency of attenuation to frequency $(\beta \propto \omega)$ can be stated and the attenuation of a given frequency will hence be the same as for its harmonics. This assumption enables the generalized Fourier series of Eq. (5) to be degenerated, taking $p=|n|$ and eliminating one sum,

$$
\mathbf{x}(\eta, \tau)=\sum_{n=-N}^{N} \hat{\mathbf{x}}_{n} \mathrm{e}^{-|n| \eta+\mathrm{j} n \tau}
$$

The Fourier base becomes

$$
\Phi_{r}=\left\{\phi_{n}(\eta, \tau)=\mathrm{e}^{-n \eta+\mathrm{j} n \tau}:[0, \infty] \times[0,2 \pi] \rightarrow C\right\}_{n=-\infty}^{\infty}
$$

and using the same inner product Eq. (6), the eigenproblem reduces to

$$
\forall n \in[-N, \ldots, N], \quad(-|n| \beta+\mathrm{j} n \omega)^{2} \mathbf{m} \hat{\mathbf{x}}_{n}+\frac{\left\langle\mathbf{f}, \phi_{n}\right\rangle}{\left\|\phi_{n}\right\|^{2}}=0
$$

Eqs. (11) or (14) are solved using a Newton-like solver in which, the terms $\left\langle\mathbf{f}, \phi_{n}\right\rangle$, i.e. the non-linear terms of Eq. (4) in the frequency domain, have to be calculated. This is done by means of an alternating frequency-time domain method adapted to the proposed formulation.

\subsection{Non-linear forces calculation}

For generic non-linearities, the frequency-domain components of the non-linear forces $\mathbf{f}(\mathbf{x}, \dot{\mathbf{x}})$ cannot generally be derived in closed form. To overcome this issue, an alternating frequencytime domain scheme is used. That is, at a given iteration of the Newton-like solver, the following process takes place.

1. Given $\left\{\hat{\mathbf{x}}_{n}\right\}_{n=-N, \ldots, N}$, the displacements and velocities are obtained in the time domain using Eqs. (12) and (9a), 
2. The time-domain form of $\mathbf{f}(\mathbf{x}, \dot{\mathbf{x}})$ then results from applying a non-linear operator (which depends on the considered non-linearity) to these displacements and velocities;

3. A projection on the Fourier harmonics is then carried out: $\left\langle\mathbf{f}, \phi_{n}\right\rangle, \forall n \in[-N, \ldots, N]$.

The interested reader might point out that such a process would, in practice, introduce some difficulties in particular due to the forms of Fourier series in Eq. (12) and of the inner product, Eq. (6), which contains an integral over infinity.

To overcome this issue, one solution is to use the AFT procedure on the underlying periodic forms of displacements and velocities and using the associated inner product. Hence, if these underlying periodic displacements and velocities are

$$
\tilde{\mathbf{x}}(\beta, \tau)=\sum_{n=-N_{h}}^{N} \hat{\mathbf{x}}_{n} \mathrm{e}^{\mathrm{j} n \tau} \quad \text { and } \quad \dot{\tilde{\mathbf{x}}}(\beta, \tau)=\sum_{n=-N_{h}}^{N} \mathrm{j} n \omega \hat{\mathbf{x}}_{n} \mathrm{e}^{\mathrm{j} n \tau},
$$

the inner product is

$$
\langle g, h\rangle_{T}=\frac{1}{2 \pi} \int_{0}^{2 \pi} g(\eta, \tau) \bar{h}(\eta, \tau) \mathrm{d} \tau,
$$

and the previous AFT scheme can be applied as is. In doing so, all variables (displacements, velocities and forces) are periodic (instead of pseudo-periodic) during the time-domain step of the calculation. While not mandatory, this modification of AFT scheme is actually consistent with the assumption that the non-conservative terms are not frequency-dependent since this implies that the decrease of non-linear forces is the same as the decrease of other variables.

In the system defined by Eqs. (11) or (14), the number of unknowns is of course superior (by two) to the number of equations. To overcome this under-determination, one need to define a set of two free variables (or parameters) which governs all remaining dependent variables. This is performed by defining a mode normalization.

\subsection{Mode normalization}

The mode normalization procedure essentially consists in defining a so-called modal amplitude. This can be done either with respect to the total energy of the system or with respect to a chosen coordinate. Here, the last approach is used; a control coordinate is chosen and the amplitude of one of its harmonics defines the modal amplitude

$$
q=q^{\Re}+\mathrm{j} q^{\Im} .
$$

It has two components real and imaginary since the mode is complex. Then each eigenvector is normalized such as

$$
\forall n \in[-N, \ldots, N], \hat{\mathbf{x}}_{n}=\boldsymbol{\psi}_{n}^{\Re} q^{\Re}+\mathrm{j} \boldsymbol{\psi}_{n}^{\Im} q^{\Im} .
$$

The complex eigenproblem (11) can then be solved with a continuation process on the modal amplitude $q$. This complex modal amplitude actually represents the two-dimensional subspace on which the motion takes place.

\section{Application on a two degrees-of-freedom system with dry-friction}

In this section, the complex non-linear modal analysis method is illustrated on the guiding example of Fig. 1a which is mathematically described by Eqs. (2). In a second step, a comparison with a direct time-integration will be proposed to validate the method. For validation purposes, all the frequency-domain analyses shown beneath have been computed with fifteen harmonics. 
Thus the approximation brought by the truncation of Fourier series is minimized while the computational efficiency increases. In practical applications (large-scale systems), fewer harmonics can be retained to achieve a satisfying compromise between accuracy and computational costs.

\subsection{Analysis of modal characteristics}

Fig. 3 shows the evolution of the eigenfrequency and modal damping in function of the modal amplitude for the first mode of the system. The asymptotic states with stuck friction point $(z=0)$ and without friction $\left(f_{N L}=0\right.$ or $\left.k_{d}=0\right)$ are shown as dashed and dash-dotted lines. As the modal amplitude increases, the transition between stick and slip leads to a decrease of the eigenfrequency. In the intermediate slip-area (amplitude around 0.06), the modal damping reaches its maximum value. This last result is typical for systems with friction whose maximum efficiency (in terms of damping) is obtained in partial slip areas.
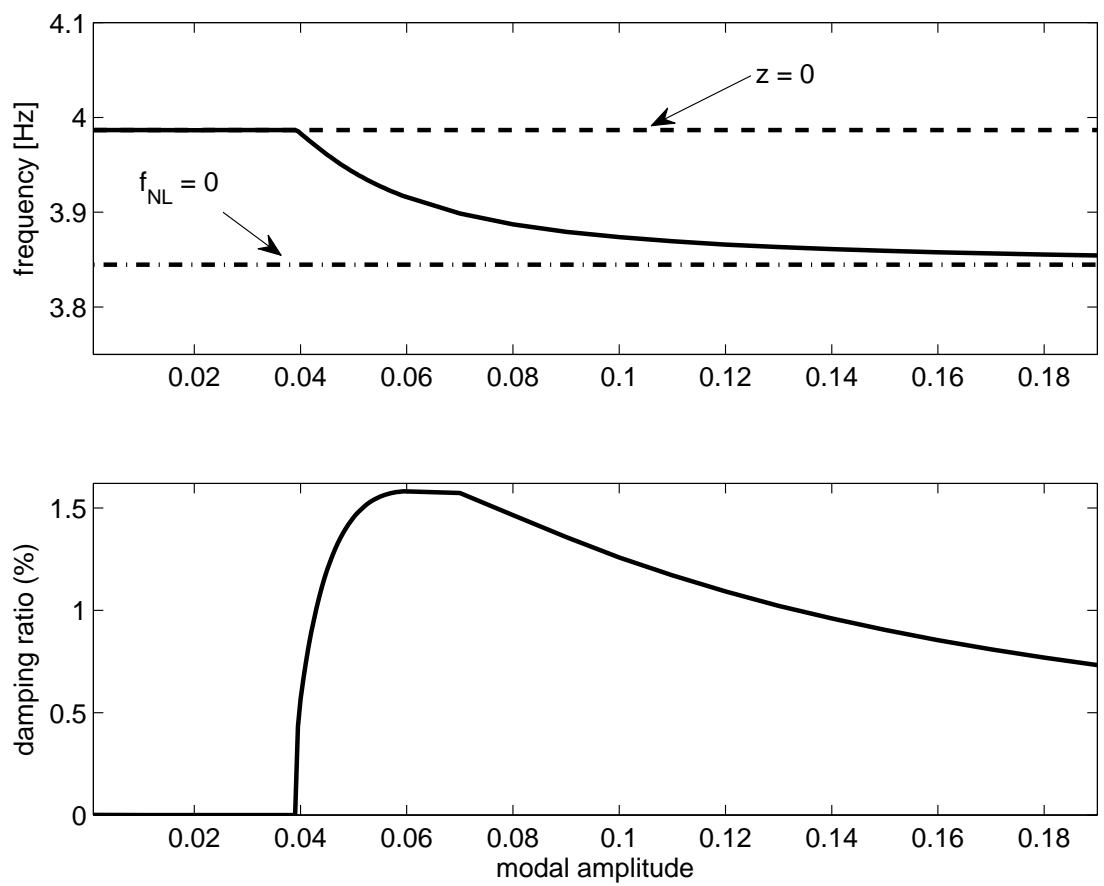

Figure 3. Eigenfrequency and modal damping as functions of the modal amplitude; (—) non-linear mode, (-- $)$ and (--) asymptotic behaviours with $z=0$ and $f_{N L}=0$ respectively.

Figs. 4 represent the two-dimensional manifolds for this mode; the displacement and velocity of the second degree of freedom are plotted as functions of the first one's and the evolution of the non-linear force is lastly shown. This typical geometrical illustration of non-linear modes clearly highlights the effects of non-linearity in comparison with the equivalent linear mode. One could in particular notice that, although surfaces of non-linear modes are tangent to those of linear modes in small amplitudes, the transition from stick to slip states of the contact point generates drastic and non-smooth changes in these surfaces both in shape and orientation. This gives information about the importance of non-linear effects as the modal amplitude changes. Change in orientation (or phase) is due to dissipative effects whereas changes in shape are due to the non-smoothness of this friction non-linearity and to a complicated dynamical behaviour occurring during alternating phases of stick and slip of the friction point. 


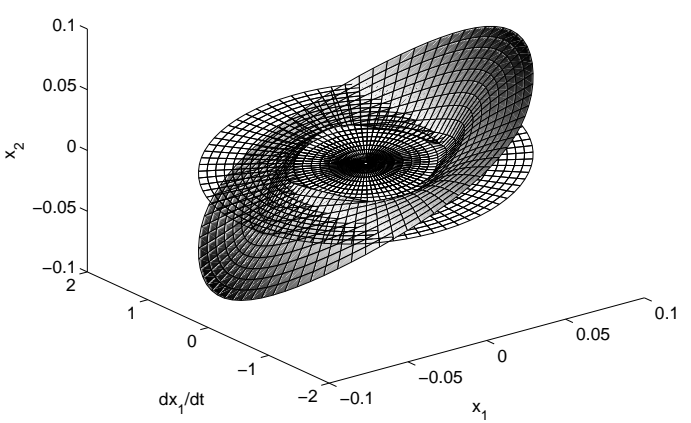

(a)

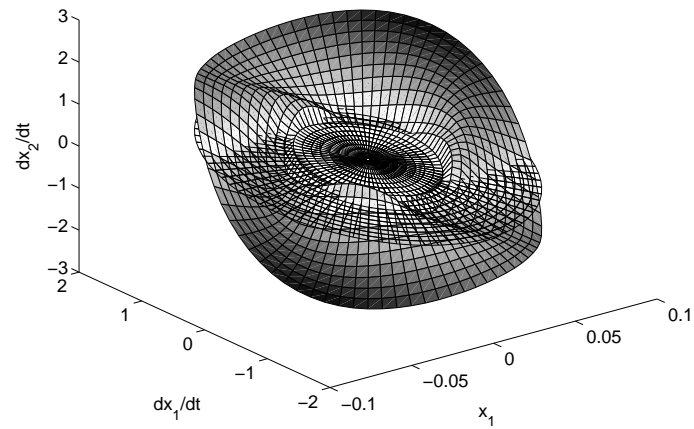

(b)

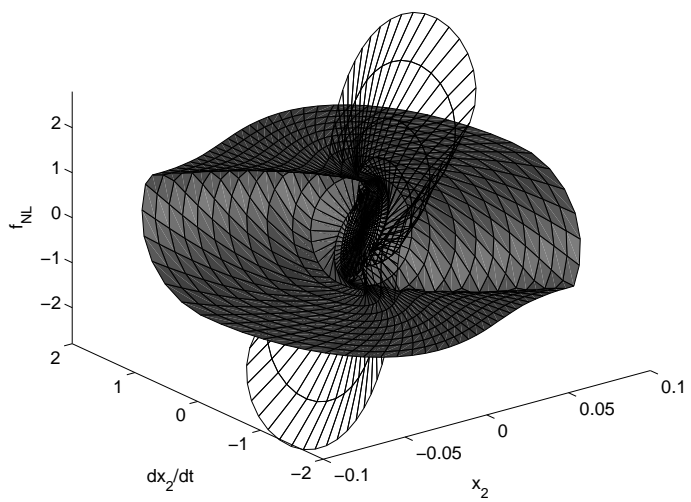

(c)

Figure 4. Manifolds of first mode: (a) and (b), displacement and velocity of 2-nd DOF and (c) evolution of non-linear force; transparent surfaces refer to the corresponding linear mode. 


\subsection{Mode stability in presence of negative damping}

Next, the effects of introducing a linear negative damping ratio in parallel to the first spring $k_{1}$ (see Fig. 1a) are investigated. This linear damping (of viscous-type here) brings a destabilizing effect which, depending on the state of the system, can be compensated by damping brought by friction. More specifically, when a negative destabilizing damping is introduced, the complex linear modes will feature eigenvalues with a positive real part and will hence be unstable. With friction, an assessment of modal damping of the non-linear modes will enable the global stability of a mode to be estimated. Also, limit cycles can be obtained.
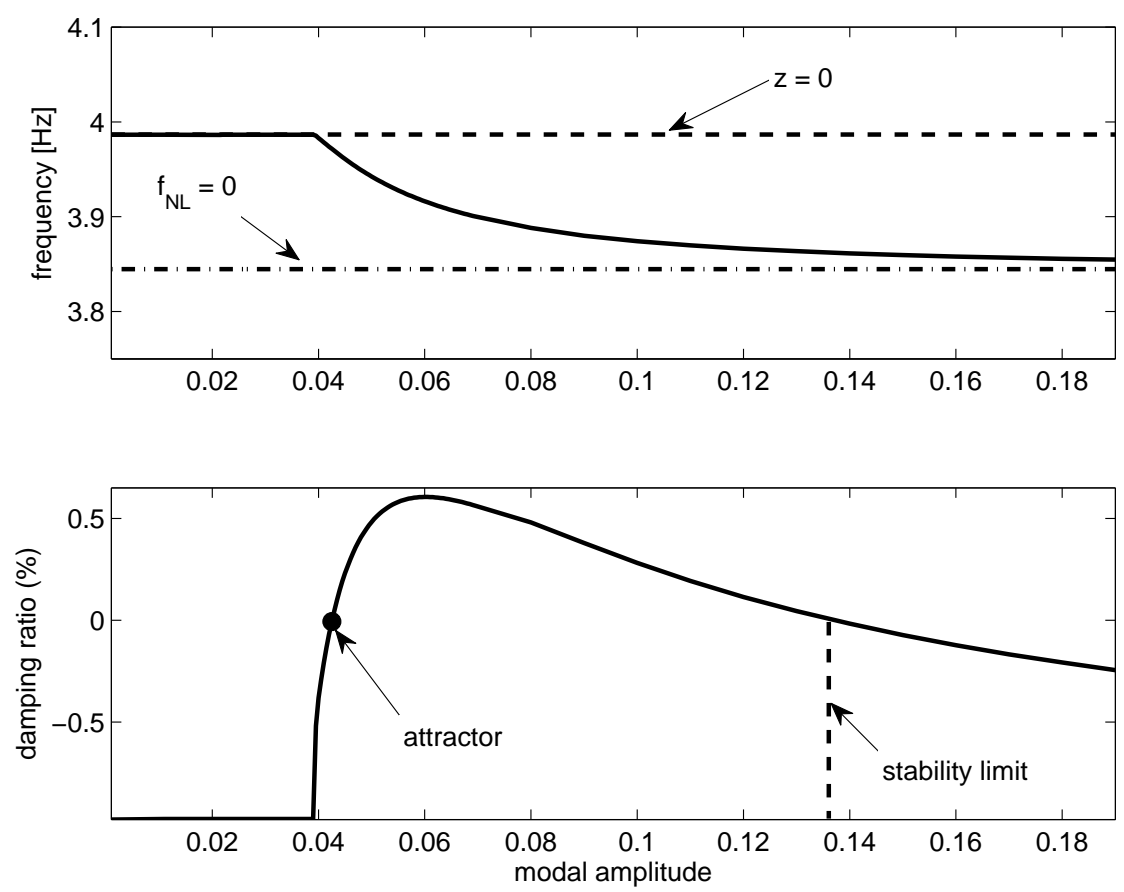

Figure 5. Case of a negative viscous damping (1\%): eigenfrequency and modal damping as functions of the modal amplitude; (-) non-linear mode, (-- $)$ and (--) asymptotic behaviours with $z=0$ and $f_{N L}=0$ respectively.

For example, Fig. 5 shows the evolution of eigenfrequency and modal damping in presence of a $1 \%$ negative damping. It is noticeable that for low or high modal amplitudes (corresponding to asymptotic configurations of stick or total slip) the mode is unstable (modal damping is negative). Conversely, in the intermediate zone of modal amplitude (and partial slip), the modal damping is positive, which means that the mode is stable.

With the view on confirming these predictions of stability, a comparison has been made between the results of a direct integration of the free system under various initial displacement conditions and the results given by non-linear modal analysis in terms of limit cycles. This comparison validates the proposed method for the calculation of free responses.

Fig. 6 gathers these results:

1. Low level initial condition: non-linear modal analysis predicts instability as well as direct integration does. Indeed, the oscillations increase from the initial value, reach a limit cycle (which corresponds to modal damping equals to zero) which is stable. This cycle is an attractor as marked in Fig. 5. 

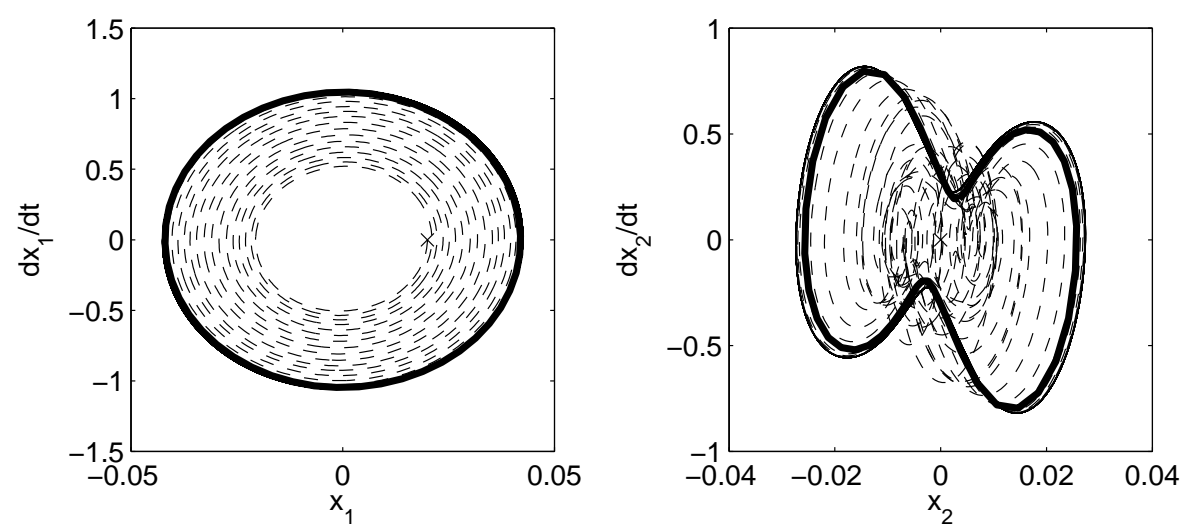

(a)
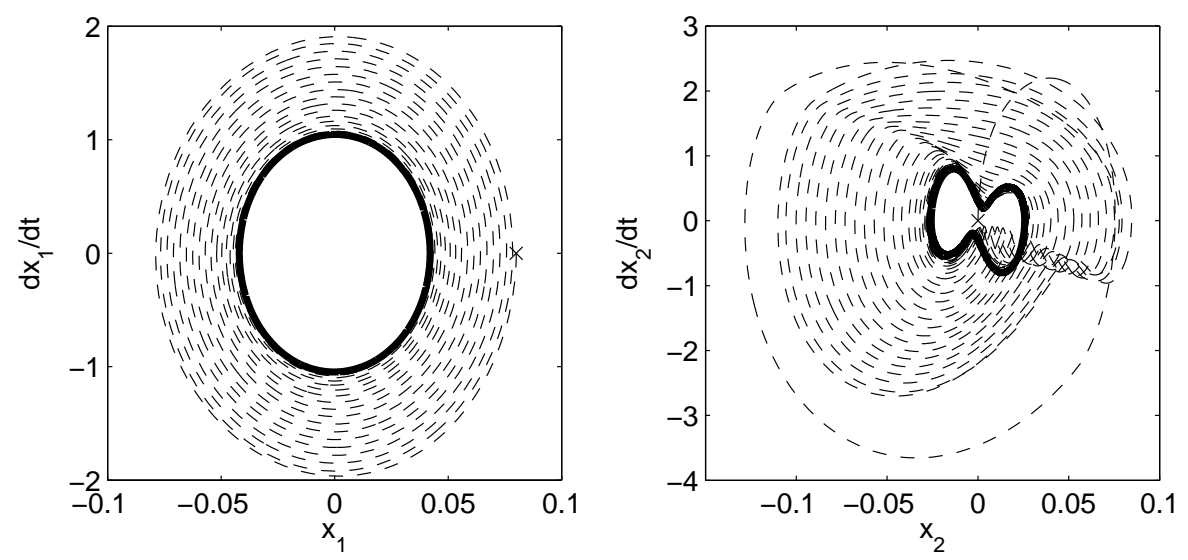

(b)
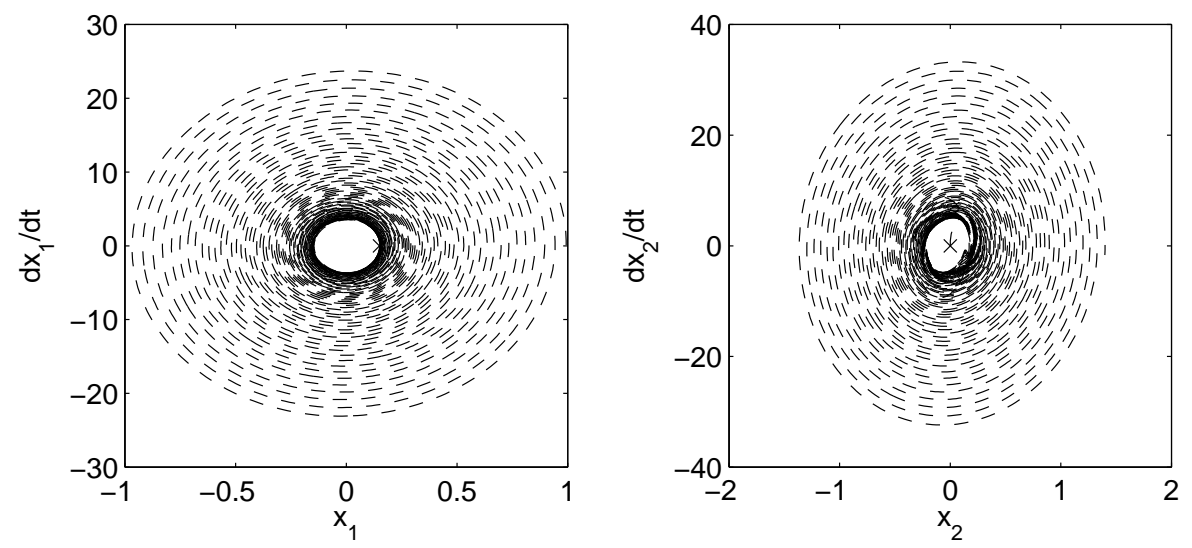

(c)

Figure 6. Predictions of stability; (-) non-linear modes and (---) direct time-integration with initial conditions $(\times)$ : (a) $x_{1}(t=0)=0.02$, (b) $x_{1}(t=0)=0.08$ and (c) $x_{1}(t=0)=0.15$. 
2. Intermediate level initial condition: modal analysis predicts a stable solution. Indeed, modal damping is positive in this range of amplitudes and direct integration shows that the level decreases from the initial condition and becomes stable on the limit cycle given by the non-linear modes.

3. High level initial condition: non-linear modal analysis predicts no stable solution. This is confirmed by time-integration since the system diverges.

\subsection{Forced responses}

Lastly, the use of non-linear modes to predict forced response is investigated. While the superposition principle does not hold for non-linear systems, non-linear modal superposition approaches have been investigated by several authors based on the so-called single non-linear mode method $[3,29,11,30]$. On the considered two degrees-of-freedom example, subject to an harmonic external force $\mathbf{p}$ of angular frequency $\Omega$, modal superposition was however not applied but the response near the resonance was approximated by a single harmonic contribution

$$
\mathbf{x}(t)=\mathfrak{R e}\left\{\boldsymbol{\psi}(q) q \mathrm{e}^{\mathrm{j} \Omega t}\right\} .
$$

Substituting, Eq. (19) in Eqs. (2) and pre-multiplying by the eigenvector $\boldsymbol{\psi}$ leads to a modal equation

$$
\left(\mu(q)\left(\omega(q)-\Omega^{2}\right)+2 \mathrm{j} \beta(q) \omega(q)\right) q=\boldsymbol{\psi}^{*}(q) \mathbf{p}
$$

in which $\mu(q)=\boldsymbol{\psi}^{*}(q) \mathbf{m} \boldsymbol{\psi}(q)$ is the generalized mass and $\lambda(q)=-\beta(q)+i \omega(q)$ is the complex eigenvalue. While the set of modal parameters $\{\lambda, \boldsymbol{\psi}\}$ functions of modal amplitude $q$ are obtained from a multi-harmonic analysis, only the fundamental harmonic was retained for the forced response synthesis. Details on the synthesis procedure can be found in Ref. [30].

Fig. 7 illustrates this forced response synthesis nearby the first resonance of the two degreesof-freedom system for several values of the forcing amplitude and shows a comparison with responses obtained by time integration. The dash curve is the so-called backbone curved, which corresponds to $q$ in function of $\omega$. While correlation is satisfying in this case even with this single mode / single harmonic contribution, convergence can be improved in general cases by considering higher order terms and other modes.

Further studies on forced response synthesis by non-linear modes would be interesting but are out of the scope of the paper. The interested reader might refer to previously mentioned references for further details.

\section{$5 \quad$ Effects of friction in turbomachinery blades dynamics}

This section describes an application of the proposed non-linear modal analysis method to an industrial case-study. This consists in studying the dynamics of a compressor blade with dryfriction at its root joint. In turbomachinery bladed-disks assemblies, dry-friction at blade root attachments is undoubtedly a major source of damping and its assessment still remains a complex issue $[24,31]$ which is difficult to address in design processes. The non-linear modal analysis approach proposed in this paper can, in this context, provide a valuable tool for analysing friction and contact effects in mechanical assemblies.

\subsection{Model description}

The finite element mesh the blade considered is shown in Fig. 8a and the first torsion mode, which deformed shape is represented in Fig. 8b, is studied. From this FE model a Craig-Bampton 


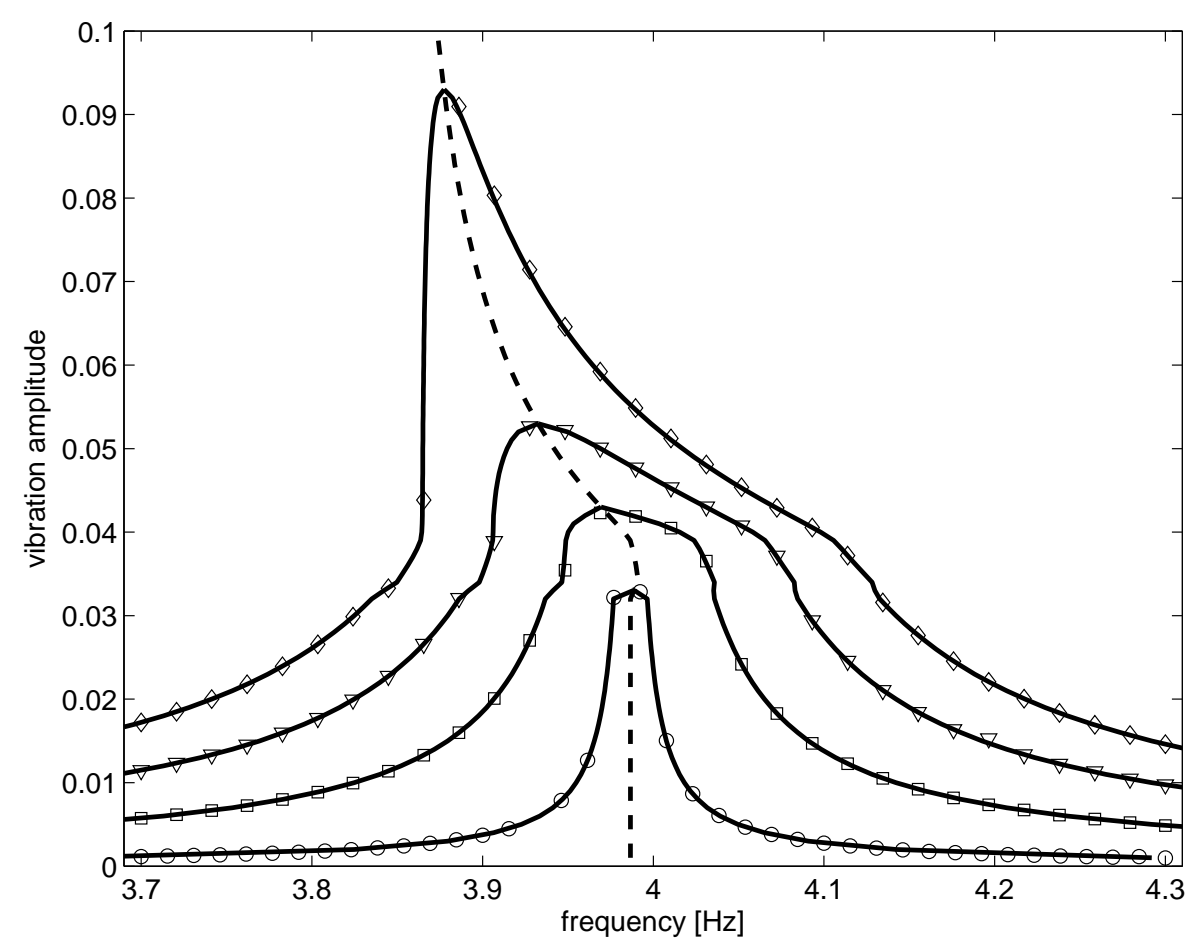

Figure 7. Forced responses synthesis with non-linear modes and comparison with time integration: solid lines, frequency responses obtained by non-linear modes for different levels of excitation; (---), backbone curve predicted by the non-linear modes; markers $(\circ, \square, \nabla, \diamond)$, responses obtained by time integration for each forcing amplitude.

reduced order model was built for which a set of $2 \times 24$ nodes at the contact interfaces plus one node (which acts as a control coordinate) at the tip trailing edge were retained. A set of normal modes (generalized degrees of freedom) was included in order to achieve a satisfying convergence of the reduced order model.

\subsection{Friction models and two-dimensional motions description}

Dry-friction is essentially a two-dimensional phenomenon. In many applications [32, 33, 34], description of surface motions and calculation of friction forces are performed by considering two (initially uncoupled) directions of motion and friction forces are determined by combining these, generally through analytical treatments. An alternative is here proposed which consists in using complex numbers to describe tangential motions. In this way, friction laws even if defined for one-dimensional motions can be used in a straightforward manner for two-dimensional motions. Hence, if $u_{T, 1}$ and $u_{T, 2}$ represent displacements in the two tangential directions, the associate complex form

$$
u_{T}=u_{T, 1}+\mathrm{j} u_{T, 2}
$$

also represents this two-dimensional tangential displacement. Similar notations are used for other quantities. As an example, if we consider the elastic Coulomb model described previously by Eqs. (3), this definition is still valid using the complex notation and absolute values are simply replaced by modulus. With this complex framework, practically any friction law (defined for one-dimensional motions) can be used as is or with little changes for two-dimensional motions.

Of interest is also to investigate on the use of friction models more representative of the tribological phenomena. In this context, one objective is to account for microscopic motions 


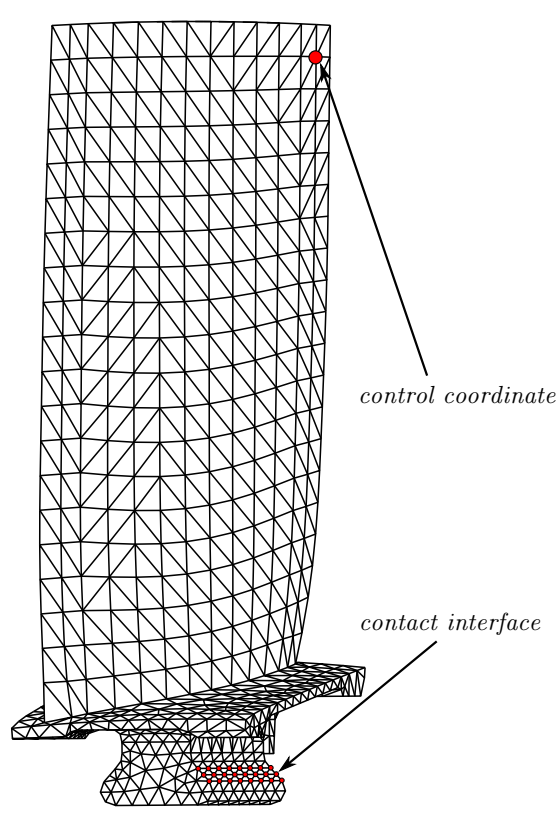

(a)

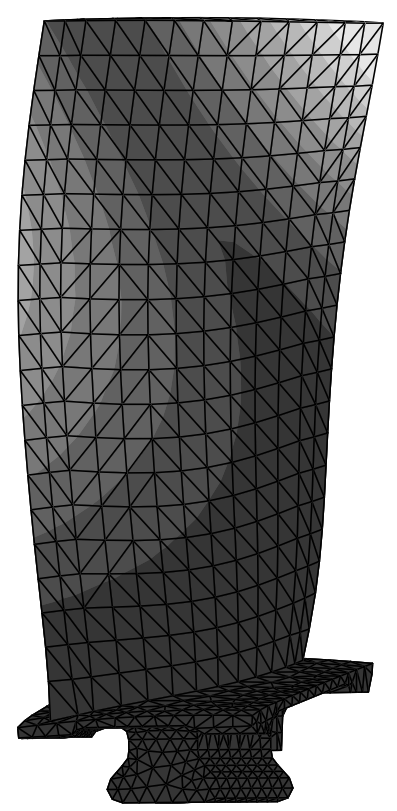

(b)

Figure 8. Compressor blade; (a) finite element mesh with retained nodes, (b) deformed shape of target mode with fixed contact interface.

(microslip) using macroscopic modelling (node-to-node contact) approaches. As this is generally not possible with a Coulomb model, a Dahl model [28] which is more sophisticated was used and compared with the Coulomb one. The Dahl model is a differential model governed by a differential equation

$$
\frac{\mathrm{d} r}{\mathrm{~d} \delta}=\sigma\left(1-\frac{r}{F_{c}} \operatorname{sgn}(\dot{\delta})\right)^{\alpha}
$$

in which, $r$ stands for the restoring force, $\delta$ is the relative tangential displacement, $F_{c}$ refers to the limit Coulomb force. The parameter $\sigma$ is an equivalent stiffness coefficient and $\alpha$ determines the shape of the stress-strain curve. Fig. 9a shows examples of hysteresis cycles in one-dimensional motion for a Dahl model. This Dahl model, even if defined for one-dimensional motions, can be directly used in two-dimensional cases if complex variables are considered. Fig. 9b illustrates trajectories of Dahl restoring force for a two-dimensional motion of the form

$$
\delta=\delta_{1} \cos t+\mathrm{j} \delta_{2} \sin t
$$

for increasing values of $\left|\delta_{1}+\mathrm{j} \delta_{2}\right|$. The limit force $F_{c}$ is equal to 1 and trajectories are clearly limited with this value (unit circle).

\subsection{First results and convergence}

In this paragraph, we show the first results on the case-study of the blade with friction at its root and discuss the convergence of the method. Fig. 10 shows the evolution of modal parameters (frequency and damping) in function of the modal amplitude. The elastic Coulomb model was used here. Several results are displayed, each corresponding to a given number of harmonics retained in the analysis.

First, concerning the modal parameters, the behaviour is quite similar (however smoother) to that in the two degrees-of-freedom model in terms of change of the natural frequency and 


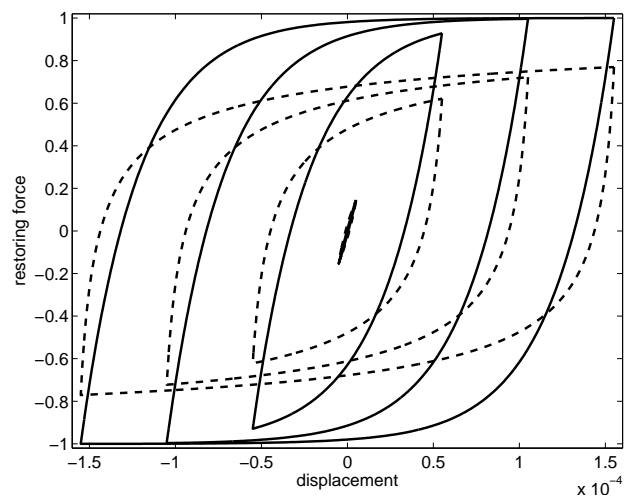

(a)

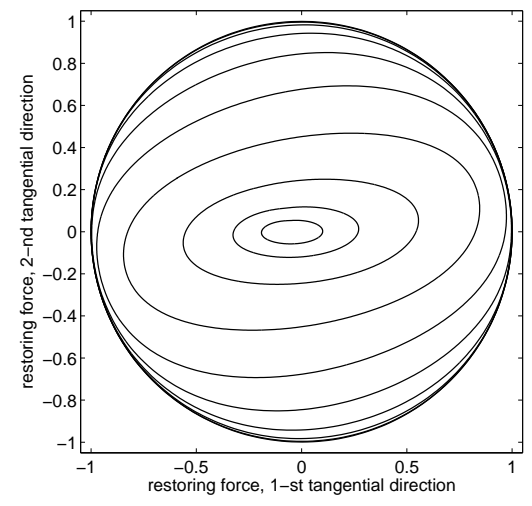

(b)

Figure 9. Friction force for a Dahl model; (a) Hysteresis cycles for (-) $\alpha=1$ and (---) $\alpha=3$ for a one dimensional tangential motion, (b) Trajectories of Dahl restoring force (for $\alpha=1$ ) for a two-dimensional tangential motion.

in the modal damping ratio. For low vibration amplitudes, there is very little slip and the natural frequency remains quite constant while the modal damping rate is almost zero; which corresponds to the asymptotic state for fixed (stuck) interfaces. In an intermediary amplitude range, the natural frequency diminishes while the damping rate increases to its maximum level; it is in this intermediary zone of sliding that friction damping is most effective. When the vibratory amplitude increases so more, it was observed that the damping rate gradually diminishes while the natural frequency still decreases asymptotically towards a sliding interfaces state.

Second, convergence of the non-linear modal analysis method is investigated on this particular example. As the basic approximation of the method is the truncation of the Fourier series, accuracy of the solution will naturally increase as the number of terms (harmonics) in the series increases. In Fig. 10, results for calculation from one to seven harmonics are represented. While one harmonics is clearly insufficient, convergence seems to be reached beyond three harmonics and the curves corresponding to five and seven harmonics are barely distinguishable. Convergence on time histories for several degrees-of-freedom was also checked and similar conclusions were found. Consequently, in the following, three harmonics were retained in order to achieve a good compromise between accuracy and computational costs. Also, in contrast with the two degrees-of-freedom system studied in section 4, few harmonics are necessary to achieve a satisfying convergence on this example. This is mostly because, due to its larger scale, this system is globally less non-linear than the latter, while the non-linearity is the same.

\subsection{Comparison of friction models and details on surface motions}

Results from non-linear modal analysis with elastic Coulomb friction model and Dahl model (for $\alpha=1$ or 2 ) are shown and compared in Fig. 11. While the global trends and values of modal parameters are quite similar between these models, the most significant difference is concerns the modal damping. Dahl models leads to higher values of modal damping. Also, the Dahl model with $\alpha=2$ gives a smoother shape to the damping curve and dissipation due to friction is also more important in small amplitude. These few remarks on the influence of friction models on non-linear modal parameters demonstrate in particular the importance of modelling microslip behaviours in such dynamical simulations.

In addition, Figs. 12 show maps of tangential displacements in the two sides of the blade root (for 24 contacts nodes, arranged in 3 columns of 8 as in the finite elements mesh) for selected 

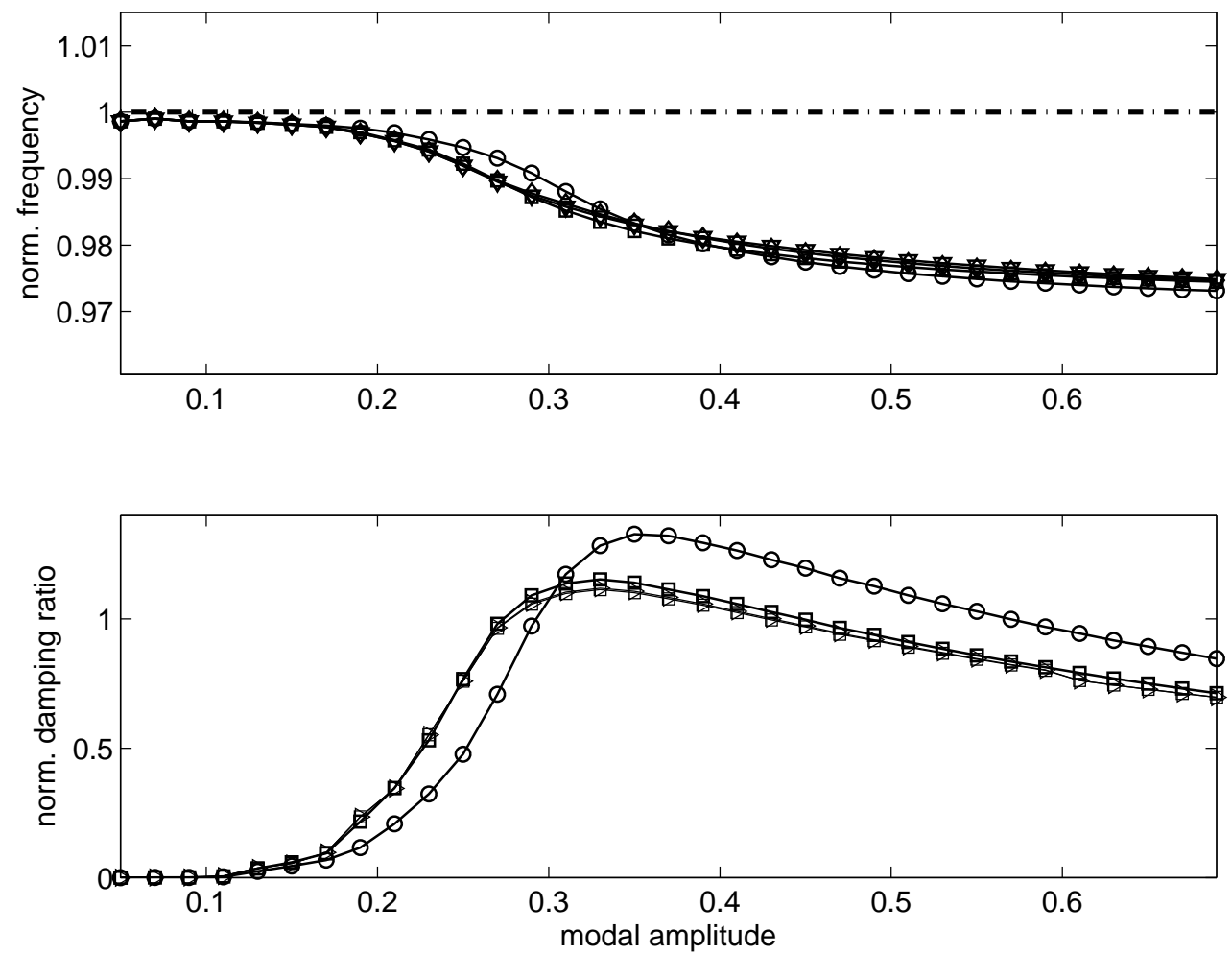

Figure 10. Non-linear modal analysis results and convergence; change in the natural frequency and in modal damping depending on modal amplitude; (-०-) one harmonic, $\left(-\square^{-}\right)$three harmonics, $\left(-\nabla^{-}\right)$five harmonics, $\left(-\diamond^{-}\right)$seven harmonics, (-:-) no slip.

values of the modal amplitude and corresponding to the calculation with the Dahl model with $\alpha=1$. Changes in tangential motions at friction interfaces are noticeable, in particular in the region of moderate modal amplitude. Indeed, comparing Figs. 12b and 12a, it seems that, as modal amplitude increases, the distribution of tangential motion gets arranged such that the displacements of all points are in the same direction. Finally, tangential motions seem to evolve less between these two last states (Fig. 12b and 12c). It is also noticeable that the distribution of slip in the two sides is globally similar (concerning the sliding nodes, not the direction of sliding) whatsoever the modal amplitude is.

\subsection{Example of parametric study}

One of the advantages of the non-linear modal analysis method proposed here is that assessment of non-linear damping is straightforward. Hence, on the considered example, the intrinsic dissipation efficiency of the frictional joint is known without any assumption on the excitation being required. This also makes parametric studies quite easy to undertake and their results to interpret, thus helping significantly the designer.

As an example, we can study the sensitivity of friction damping with respect to the coefficient of friction. On the same example, in Fig. 13 we show the change in modal data (natural frequency and modal damping) for several friction coefficient values using the Dahl model. First, it can be seen that the lower the friction coefficient is, the lower the sliding threshold will be. Second, the difference between the limit values of this threshold is quite large, which highlights the sensitivity of damping mechanisms to changes in the contact parameter. However, it can also be seen that maximum damping rate remains practically constant. 

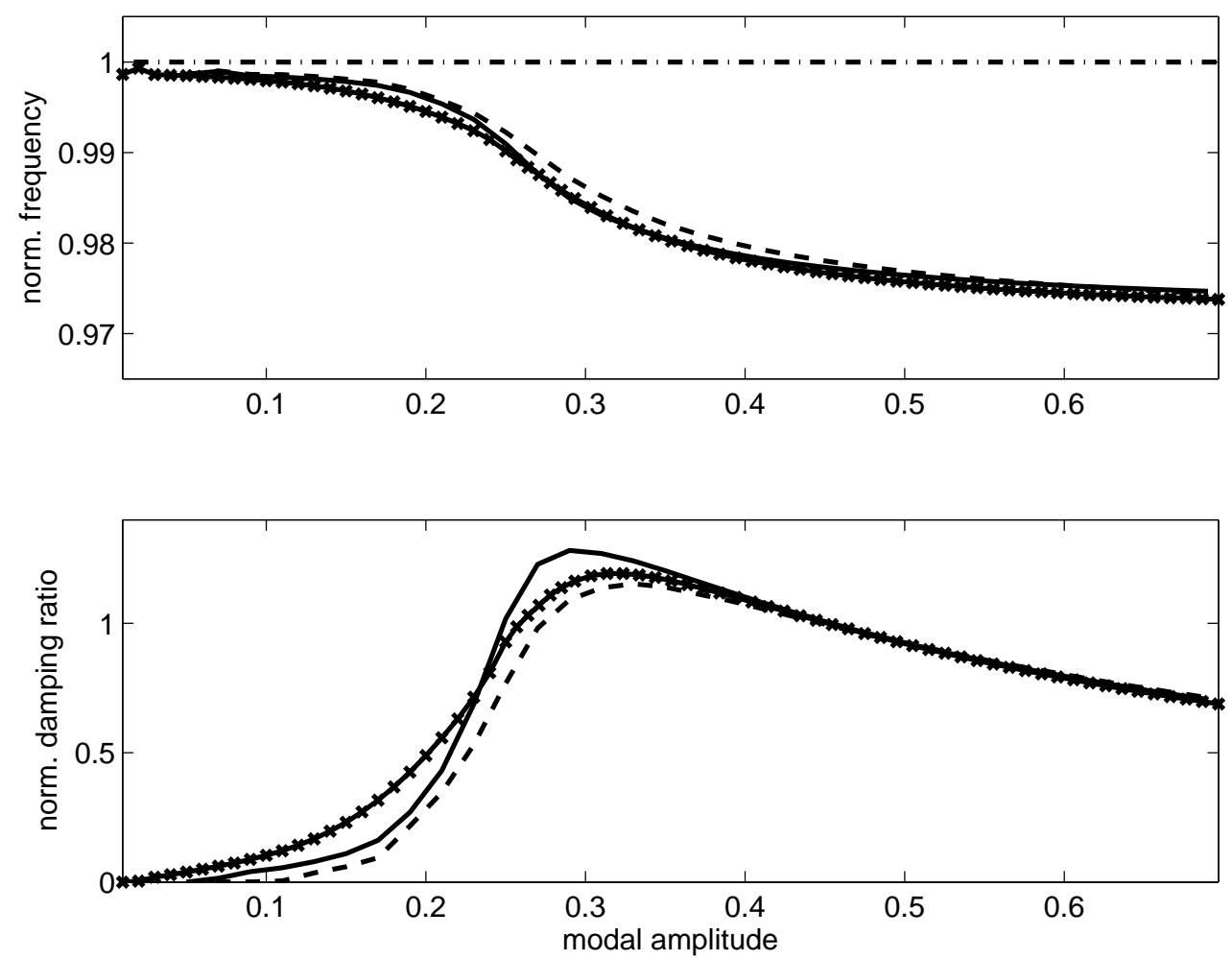

Figure 11. Effects of friction model on modal parameters; (- - -) Coulomb model, ( - ) and $(\mathbf{-} \times \mathbf{-})$ Dahl model with $\alpha=1$ and $\alpha=2$ respectively, (---) no slip.

Such parametric studies, which are necessary in the design process of turbomachinery bladings (as for friction damping devices), are quite easy to undertake and the results are also easy to interpret with the presented approach since the level of uncertainty is reduced to a minimum. In particular, the proposed modal approach makes it possible to evaluate the sensitivity of the damping properties with respect to any design (or environment) parameters without any assumptions on the excitation. In the future, considering other environment variables, such as fretting-wear [35, 36], as additional modal parameters can be interesting.

\section{Conclusions}

A non-linear modal analysis method has been proposed; it is based on the concept of complex non-linear modes and on a frequency-domain formulation of the eigenproblem associated with the non-conservative autonomous dynamical system. The eigensolutions are thought in the form of generalized Fourier series which allows the treatment of non-conservative non-linearities. Although the developments focus on the case of systems with frequency-independent dissipative terms, the general form of the generalized Fourier series should be applicable to other class of systems.

Thus, beyond frequencies and mode shapes, this method supplies modal damping rates which, as other modal quantities, depend on the energy of the system. This approach appears particularly relevant for systems featuring dissipative non-linearities and which cannot be represented by an equivalent conservative (non-linear) system. Examples addressed in this work mostly deal with friction non-linearities but the method is applicable to any generic type of non-linearities. 


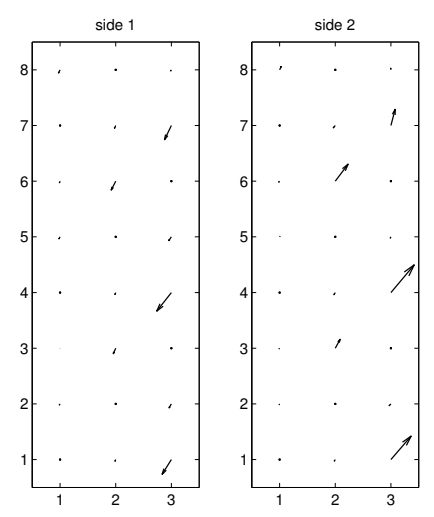

(a)

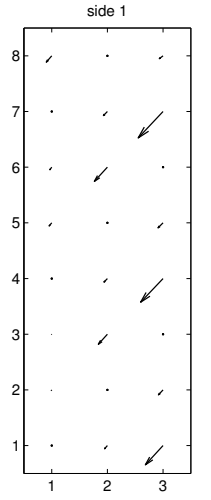

(b)

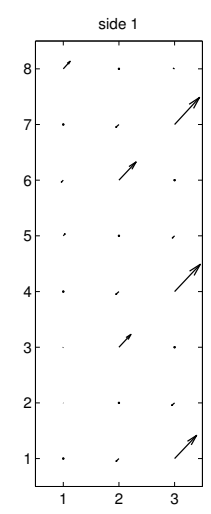

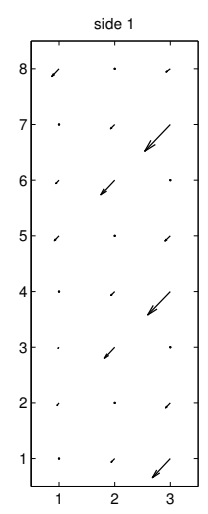

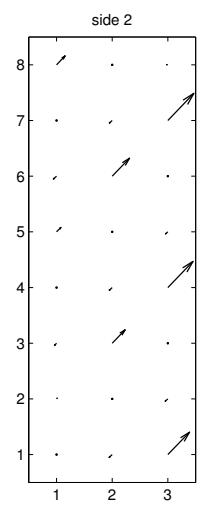

(c)

Figure 12. Maps of tangential displacements; (a) modal amplitude 0.19 and scale $\times 1000$, (b) modal amplitude 0.29 and scale $\times 250$, (c) modal amplitude 0.59 and scale $\times 100$.

Large-scale systems can also be treated as demonstrated in an industrial application dealing a turbomachinery compressor blade with dry-friction at its root interface. On this example, a detailed description of the treatment of two-dimensional frictional motion through complex variables is given for two particular friction models, namely Coulomb and Dahl ones.

Within this framework, many future investigations and developments can be considered among which:

- analyses of stability and bifurcations;

- coupling of non-linear dynamics with other contact issues such as the effects of frettingwear which can be efficiently analysed if represented as an additional modal parameter;

- in the scope of turbomachinery applications, multi-physic coupling and non-linear aeromechanical analyses can be imagined.

\section{Acknowledgement}

Thanks go to Snecma for its technical and financial support. This work takes place in the framework of the MAIA mechanical research and technology program sponsored by CNRS, ONERA and SAFRAN Group. We also thank Mathias Legrand for valuable and constructive discussions.

\section{References}

[1] R. M. Rosenberg, The normal modes of nonlinear n-degrees-of-freedom systems, Journal of applied Mechanics 30 (1962) 595-611.

[2] R. Rand, A direct method for nonlinear normal modes, International journal of Non-linear Mechanics 9 (1974) 363-368. doi:10.1016/0020-7462(74)90021-3.

[3] W. Szemplinska-Stupnicka, "Non-linear normal modes" and the generalized Ritz method in the problems of vibrations of non-linear elastic continuous systems, International Journal of Non-Linear Mechanics 18 (2) (1983) 149-165. doi:10.1016/0020-7462(83)90042-2. 

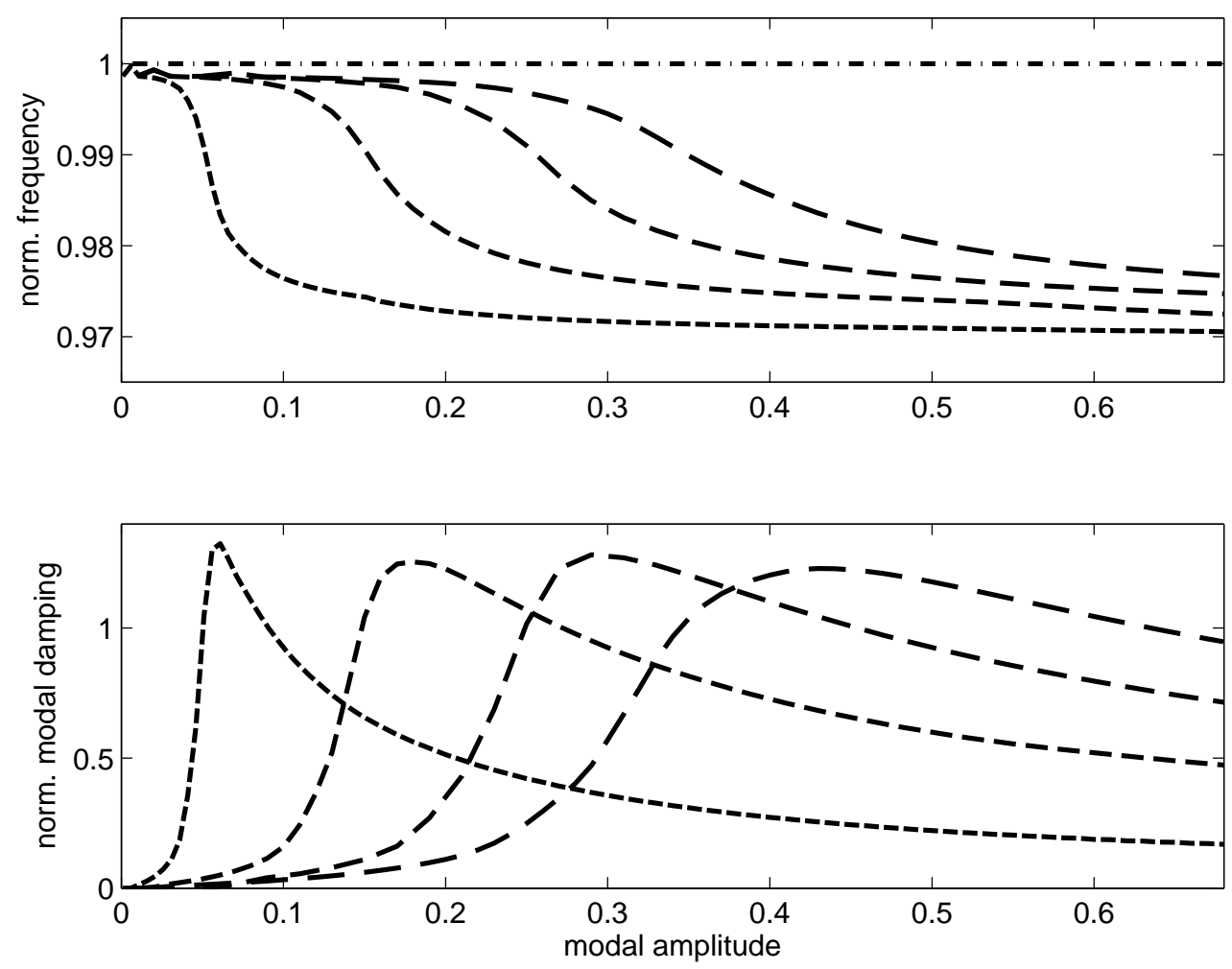

Figure 13. Influence of the friction coefficient on modal parameters; (---) $\mu=0.1,(\mathbf{- - \boldsymbol { - }}) \mu=0.3$, $(\boldsymbol{-}) \mu=0.5,(\boldsymbol{Z} \boldsymbol{-}) \mu=0.7,(-\cdot-)$ no slip.

[4] S. W. Shaw, C. Pierre, Normal modes for non-linear vibratory systems, Journal of Sound and Vibration 164 (1) (1993) 85-124. doi:10.1006/jsvi.1993.1198.

[5] A. F. Vakakis, L. I. Manevitch, Y. V. Mikhlin, V. N. Pilichuk, A. A. Zevin, Normal Modes and Localization in Nonlinear Systems, Wiley series in nonlinear science, 1996.

[6] L. Jézéquel, C. H. Lamarque, Analysis of non-linear dynamical systems by the normal form theory, Journal of Sound and Vibration 149 (3) (1991) 429-459. doi:10.1016/0022-460X (91)90446-Q.

[7] A. H. Nayfeh, On direct methods for constructing nonlinear normal modes of continuous systems, Journal of Vibration and Control 1 (4) (1995) 389-430. doi:10.1177/107754639500100402.

[8] A. F. Vakakis, L. I. Manevitch, O. Gendelman, L. Bergman, Dynamics of linear discrete systems connected to local, essentially non-linear attachments, Journal of Sound and Vibration 264 (2003) 559-577. doi:10.1016/S0022-460X (02)01207-5.

[9] D. Laxalde, F. Thouverez, J.-J. Sinou, Dynamics of a linear oscillator connected to a small strongly non-linear hysteretic absorber, International Journal of Non-Linear Mechanics 41 (8) (2006) 969-978. doi:10.1016/j.ijnonlinmec.2006.09.002.

[10] S. Setio, H. Setio, L. Jézéquel, Modal analysis of nonlinear multi-degree-of-freedom structure, The International Journal of Analytical and Experimental Modal Analysis 7 (2) (1992) 75-94. 
[11] Y. H. Chong, M. Imregun, Development and application of a nonlinear modal analysis technique for mdof systems, Journal of Vibration and Control 7 (2) (2001) 167-179. doi:10.1177/107754630100700202.

[12] G. Kerschen, M. Peeters, J. C. Golinval, A. F. Vakakis, Nonlinear normal modes, part I: A useful framework for the structural dynamicist, Mechanical Systems and Signal Processing. doi:10.1016/j.ymssp.2008.04.002.

[13] E. Pesheck, C. Pierre, S. W. Shaw, A new galerkin-based approach for accurate non-linear normal modes through invariant manifolds, Journal of Sound and Vibration 249 (5) (2002) 971-993. doi:10.1006/jsvi.2001.3914.

[14] B. Cochelin, N. Damil, M. Potier-Ferry, Asymptotic-numerical methods and pade approximants for non-linear elastic structures, International Journal for Numerical Methods in Engineering 37 (7) (1994) 1187-1213. doi:10.1002/nme.1620370706.

[15] R. Arquier, S. Bellizzi, R. Bouc, B. Cochelin, Two methods for the computation of nonlinear modes of vibrating systems at large amplitudes, Computers \& Structures 84 (24-25) (2006) 1565-1576. doi:10.1016/j.compstruc.2006.01.011.

[16] M. Peeters, R. Viguié, G. Sérandour, G. Kerschen, J.-C. Golinval, Nonlinear normal modes, part II: Toward a practical computation using numerical continuation techniques, Mechanical Systems and Signal Processing. doi:10.1016/j.ymssp.2008.04.003.

[17] C. Pierre, A. A. Ferri, E. H. Dowell, Multi-harmonic analysis of dry friction damped systems using an incremental harmonic balance method, Journal of Applied Mechanics 52 (1985) 958-964.

[18] A. Cardona, T. Coune, A. Lerusse, M. Géradin, A multiharmonic method for non-linear vibration analysis, International Journal for Numerical Methods in Engineering 37 (9) (1994) 1593-1608. doi:10.1002/nme.1620370911.

[19] S. Nacivet, C. Pierre, F. Thouverez, L. Jézéquel, A dynamic lagrangian frequency-time method for the vibration of dry-friction-damped systems, Journal of Sound and Vibration 265 (2003) 201-219. doi:10.1016/S0022-460X (02) 01447-5.

[20] T. M. Cameron, J. H. Griffin, An alternating frequency/time domain method for calculating the steady-state response of nonlinear dynamic systems, Journal of Applied Mechanics 56 (1989) 149-154.

[21] J. H. Griffin, A review of friction damping of turbine blade vibration, International Journal of Turbo and Jet Engines 7 (1990) 297-307.

[22] M. Berthillier, C. Dupont, R. Mondal, J. J. Barrau, Blades forced response analysis with friction dampers, Journal of Vibration and Acoustics 120 (1998) 468-474.

[23] E. P. Petrov, Analytical formulation of friction interface elements for analysis of nonlinear multi-harmonic vibrations of bladed disks, Journal of Turbomachinery 125 (2) (2003) 364. doi:10.1115/1.1539868.

[24] D. Charleux, C. Gibert, F. Thouverez, J. Dupeux, Numerical and experimental study of friction damping in blade attachments of rotating bladed disks, International Journal of Rotating MachineryArticle ID 71302, 13 pages. 
[25] D. Laxalde, F. Thouverez, J. P. Lombard, Vibration control for integrally bladed disks using friction ring dampers, in: Proceedings of ASME Turbo Expo, no. GT2007-27087, Montréal, Canada, 2007.

[26] J. G. Marshall, M. Imregun, A review of aeroelasticity methods with emphasis on turbomachinery applications, Journal of Fluids and Structures 10 (1996) 237-267.

[27] R. E. Kielb, J. W. Barter, J. P. Thomas, K. C. Hall, Blade excitation by aerodynamic instabilities - a compressor blade study, in: Proceedings of ASME Turbo Expo, Atlanta, Georgia, USA, 2003.

[28] P. R. Dahl, Solid friction damping of mechanical vibrations, AIAA Journal 14 (12) (1976) $1675-1682$.

[29] S. Setio, H. D. Setio, L. Jézéquel, A method of non-linear modal identification from frequency response tests, Journal of Sound and Vibration 158 (3) (1992) 497-515. doi:10.1016/0022-460X (92) 90421-S.

[30] C. Gibert, Fitting measured frequency response using non-linear modes, Mechanical systems and signal processing 17 (1) (2003) 211-218.

[31] E. P. Petrov, D. J. Ewins, Effects of damping and varying contact area at blade-disk joints in forced response analysis of bladed disk assemblies, Journal of Turbomachinery 128 (2) (2006) 403-410. doi:10.1115/1.2181998.

[32] K. Y. Sanliturk, D. J. Ewins, Modelling two-dimensional friction contact and its application using harmonic balance method, Journal of Sound and Vibration 193 (2) (1996) 511-523. doi:10.1006/jsvi.1996.0299.

[33] C. H. Menq, B. D. Yang, Non-linear spring resistance and friction damping of frictional constraint having two-dimensional motion, Journal of Sound and Vibration 217 (1) (1998) 127-143. doi:10.1006/jsvi.1998.1739.

[34] F. Xia, Modelling of a two-dimensional coulomb friction oscillator, Journal of Sound and Vibration 265 (5) (2003) 1063-1074. doi:10.1016/S0022-460X (02)01444-X.

[35] L. Salles, L. Blanc, F. Thouverez, A. Gouskov, Analysis of a bladed disk with friction and fretting-wear in blade attachments, in: Proceedings of ASME Turbo Expo, no. GT200851112, Berlin, Germany, 2008.

[36] D. Laxalde, L. Salles, L. Blanc, F. Thouverez, Non-linear modal analysis for bladed disks with friction contact interfaces, in: Proceedings of ASME Turbo Expo, no. GT2007-50860, Berlin, Germany, 2008. 